\title{
The Effectiveness of Calcaneal Drilling for Treatment of Resistance Cases of Plantar Heel Pain \\ ${ }^{1}$ Mohammed SH. Al-Edanni ${ }^{2}$ Basim S. Abdullah
}

\section{ABSTRACT}

Background: Plantar heel pain is a clinical syndrome characterized by pain and tenderness beneath the heel which is typically worse in the morning and improves after the first few steps in the day. It is a common and frequently disabling clinical complaint that may be caused by a broad spectrum of osseous or soft tissue disorders

Objective: To evaluate the effectiveness of an operation of multiple drilling of calcaneum for resistant plantar heel pain syndrome.

Methods: During the period from November 2012 to August 2016, 17 patients (17heels) were enrolled in a cohort clinical study at the orthopedic unit in AL-Sheikh Zayed and AlWassity Hospitals.

Result: Drilling of the calcaneum is a simple procedure achieving $70.6 \%$ cure in resistant ases with few complications provided good patient selection was done.

Conclusion: Abnormalities in the intraosseous pressure within the calcaneum are a possible cause of plantar heel pain in some patients who failed to respond to conservative treatment

Key words: Plantar heel pain, calcaneal drilling, intraosseous pressure.

${ }^{1}$ Correspondence author: Assistant Professor, FICMS (ortho). Orthopedic Surgeon in AlKindy College of Medicine

${ }^{2}$ Orthopedic Surgeon in Al-Shaheed Al-Sadeer Hospital. FICMS (ortho)

Received at 3/4/2017

Accepted at 20/12/2017

\section{INTRODUCTION}

$\mathrm{P}$ lantar heel pain is a clinical syndrome characterized by pain and improves after the first few steps in the day ${ }^{(1)}$. It is a common and frequently disabling clinical complaint that may be caused by a broad spectrum of osseous or soft tissue disorders

Although a variety of terms used to describe the syndrome, (heel spur syndrome, Calcaneodynia) still the name of plantar painful heel in used deliberately in the preference to any other more of precise etiological diagnosis, since the cause of this definite clinical entity still remain uncertain ${ }^{(2)}$

\section{Etiology:}

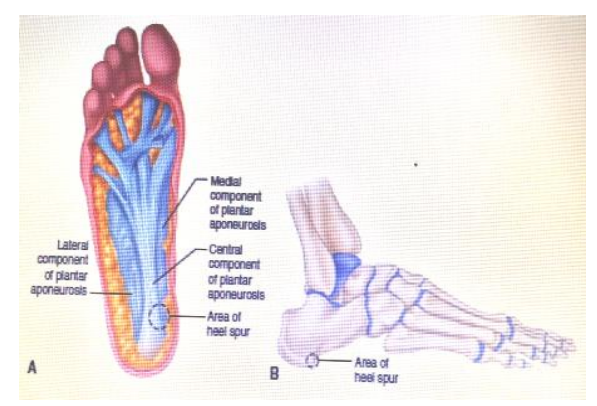

Figure (1): Painful heel. Most common location heel pain. ${ }^{(4)}$

Although roentgenograms of the symptomatic heel reveals calcaneal spur in $50 \%$ of patients, it is well accepted that the
1. Heel pad degeneration

2. Windlass mechanism of plantar fascia

3. Neurogenic etiology

4. Increased intra osseous pressure. ${ }^{(3,4,5)}$

\section{Risk factors}

1. Over use and repetitive, obesity, sudden weight gain, Age.

2. Abnormal foot such as pes cavus, pes planus over pronated hind foot,

3 . Tightness and weakness in the gastrosoleus, Achilles tendon and foot intrinsics

4. Elderly (over 70y) also at risk because of weakness in muscles,

5. Type of employment with prolonged standing and walking. ${ }^{(6,7)}$

spur by itself is not the etiological factor since $16-25 \%$ of normal population had spurs and $46 \%$ of patients with painful heel had no spurs and $50 \%$ of patients with bilateral spurs had pain in only one heel ${ }^{(8)}$, it is only a preexisting oversized medial calcaneal tuberosity that are more common as peoples age, and its position is inside the origin of the short flexors deep to the plantar fascia and weight bearing part of the calcaneum ${ }^{(3,8,9)}$.(Fig.1) 


\section{METHODS}

During the period from November 2012 to August 2016, 17 patients (17heels) were enrolled in a cohort clinical study at the orthopedic unit in AL-sheikh Zayed and Alwassity Hospitals.

They were 8 women and 9 men ranging in age between 30 to 53 year (mean 42.5 year). 14 of them above the age of 40 year, all patients complained of diffuse plantar heel pain with duration between 9 months and 10 years (mean 2.5 years), all women were housewives and men involved in jobs which needs standing for long duration, 11 cases $(64.7 \%)$ were the right side and 6 cases $(35.3 \%)$ are left side.

All patients have diffuse tenderness over the heel with pain on standing none have paresthesia or pain on tapping the posterior tibial nerve and all patients have normal foot anatomy (neither flat nor cavus). Blood tests (ESR, blood sugar and Serum uric acid) were done for all patients and were within normal range, no patient had evidence of connective tissue disease or any other intercurrent illnesses.

Lateral $x$-ray of both heels in standing position was taken (Fig.2), six patients showed no calcaneal spur, 10 showed unilateral spur on the painful side for which operation was done .None of them showed abnormal pathologies on the plain $\mathrm{x}$-ray or abnormal biomechanical alignment of the foot bones. The heel pad thickness was between $(7-11 \mathrm{~mm})$ mean of $9 \mathrm{~mm}$ (normal range is $9.9 \mathrm{~mm}$ for $20-40$ year and 11.5for 41-60 year). X-ray of the knee and lumbar spine were taken to exclude osteoarthritis

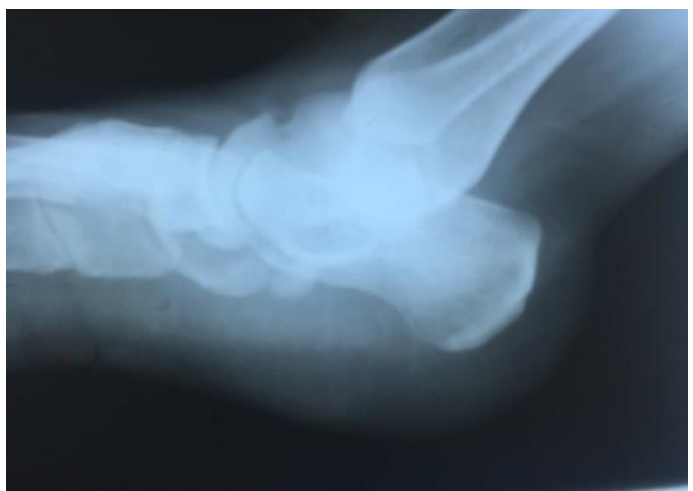

Figure 2 : Pre-oparative X ray

Our patients were selected among 60 patients who were subjected to 3 courses of conservative treatment. The treatment includes rest, stretching exercise, NSAID, heel cups and local steroid injection for all. Forty three patients improved and were excluded, and only the 17 patients who did not improve were included, and reassessed with thorough history and clinical examinations and were assessed for their symptoms by the modified Maryland foot scoring system ${ }^{(7)}$. It consists of 12 points, (5 for pain, 4 for function and 3 for limping), the points increased with the severity of symptoms (12 for most severe and 0 for no symptoms):

1. Pain

0 None (including with sport activity).

1 Slight (no change in activity of daily living or work).

2 Mild (minimal change in activity of daily or work).

3.moderate (substantial decrease in activity of daily living or work, with frequent use of analgesics).

4 Marked (pain during minimal activity, more frequent use of stronger analgesics).

5 Disabling (unable to work or shop).

\section{Function (walking distance).}

0 Unlimited.

1 -Slight limitation.

2 -Moderate limitation (2-3 blocks; 200300 meter).

3-Severe limitation (1 block; 100 meter).

4- Indorse only.

\section{Limping:}

0 - None

1- Slight

2 -Moderate.

3- Severe.

The patients selected for surgery had scores of at least 7 points (moderate pain, moderate function limitation, limping) their range was between 7 and 12 .

Patient selection for surgery was done according to inclusion and exclusion criteria to avoid improper selection for the surgical procedure, these include:

Exclusion criteria

1. History of previous surgery.

2.Systemic diseases (seronegative arthropathy, RA,.)

3.--'Neurologic, vascular or metabolic diseases including DM and gout.

4. Definite osteoarthritis of the knee joint or lumbo-sacral spine.

Surgery was performed under general anesthesia without tourniquet control for 
the purpose of measuring the intra osseous pressure, a longitudinal $5 \mathrm{~cm}$ lateral skin incision was done 1 inch below the tip of the lateral malleolus extending anteriorly from the peroneal trochlea to the lower insertion of the tendo achillis to the calcaneum posteriorly (Fig.3), only the skin was opened by sharp dissection, isolate and ligate the communicating veins, carefully isolate the sural nerve and the small saphenous vein and protect them, now the flat lateral surface of the calcaneum exposed with the peroneal tendons enclosed in the inferior peroneal retinaculum in the upper part of the wound and the fleshy belly of the abductor digiti minimi in its lower part. Leaving the periosteum intact until the intraosseous pressure measurement in the calcaneum is completed by using by using a simple device (central venous pressure measurement set ) consisting of a plastic tube with an end filter and clamp a white scale graded from 2 to $29 \mathrm{~cm}$ with a red bar slides over the scale up and down and gutter which can adapt tube a wide bore thin wall siliconed needle of $2 \mathrm{~cm}$ length and (180G) a three way valve adapter and

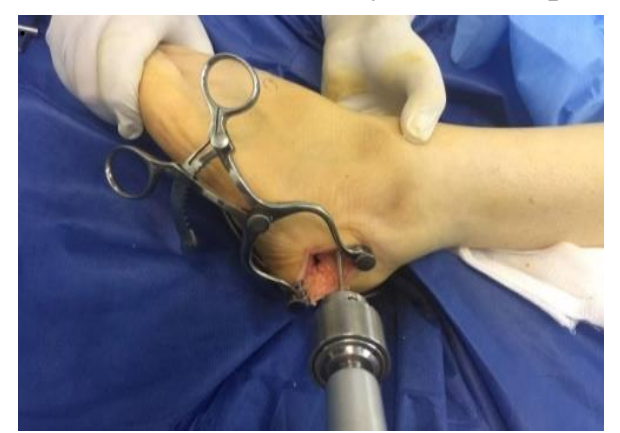

Figure 4: Drilling

\section{RESULTS}

The study sample consists of seventeen patients (17heels), 9 males (52.9\%) and 8 females $(47.1 \%)$, mean age 42.5 year, all females were housewives, regarding male patients 2 males (22.2\%) were employee, The nature of pain was throbbing in 9 $(52.9 \%)$ and searing or pricking in 8 $(47.1 \%)$ morning pain was present in all, All patients had diffuse tenderness over the plantar heel area and pain on standing none complained of paresthesia and numbness in the medial aspect of foot and heel. Blood tests were normal for all patients, $\mathrm{x}$-rays revealed bony spur in 10 $(58.8 \%)$, spurs were absent in 7 patients aplastic giving set for intravenous fluid with one unit $(500 \mathrm{cc})$ of isotonic normal salin. Then after intraosseous pressure measurement, open the periosteum by sharp dissection and elevate it by periosteal elevator to expose the bone, with the use of electrical drill or preferably a hand drill using $2 \mathrm{~mm}$ drill bit perform 5 holes through the calcaneum from lateral cortex to the medial cortex in a horizontal manner $1 \mathrm{~cm}$ above the inferior edge of the bone and the holes separated (Fig.4). Only skin was closed without drain (Fig.5), the foot wrapped with compressive bandage, elevation for 72 hours, complete weight bearing allowed 3 days postoperatively

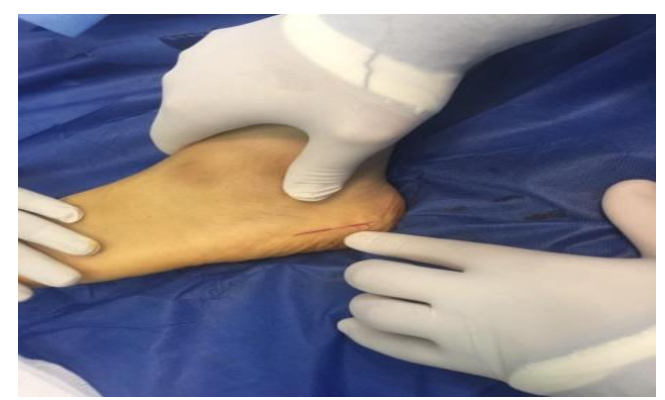

Figure 3 skin incision

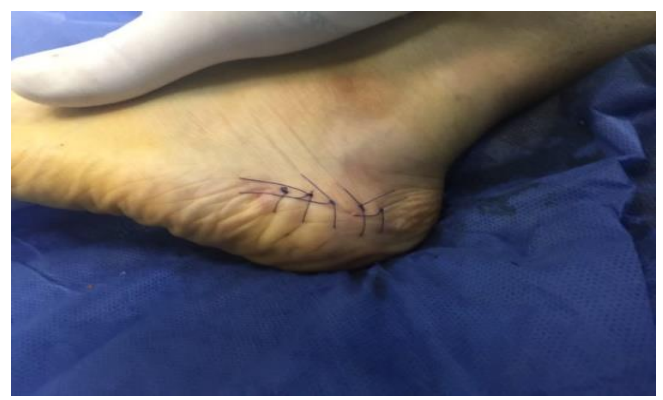

Figure 5: Wound suturing

2 males $(22.2 \%)$ were labors and 5 males (55.4\%) with miscellaneous jobs. Regarding the body mass index 5 patients $(29.4 \%)$ were obese $(\mathrm{BM}>=30), 8$ $(47.11 \%)$ were overweight $(\mathrm{BM}=25-29.9)$ and 4 patients $(23.5 \%)$ were ideal (BMI=20-24.9).

14 patients $(82.4 \%)$ had pain at rest and night pain was sound in 15 patients $(88.2 \%)$.

$(41.2 \%)$ the heel pad thickness was between $(7-11 \mathrm{~mm})$ mean $9.3 \mathrm{~mm}$.

The modified Maryland foot score (MFS) was between 7-12 points with mean of 10 points before surgery. The frequency distribution of the study sample by the components of MFS was: No pain 
one patient $(5.9 \%)$, moderate $5(29.4 \%)$ and disabling pain in 11 patients $(64.7 \%)$.

In this study 12 feet $(70.6 \%)$ were cured at the end of the 6 months follow up (one foot $(8.3 \%)$ at the end of one month, 4 feet $(33.3 \%)$ at the end of three months and 7 feet $(58.4 \%)$ at the end of six months). Five feet $(29.4 \%)$ were still complaining of heel pain at the end of follow up. Regarding the complications, one foot (5.9\%) developed sural nerve injury with neuroma formation and two feet $(11.8 \%)$ superficial skin infection wound healing was within the normal period for the lower limb without scar problems and all feet wear ordinary shoes post operatively. None of the patients developed bleeding during or after surgery the average time of the patients stay out of job was between 34 weeks and all of them returned to job after one month.

\section{DISCUSSION}

Plantar heel syndrome is a common clinical problem yet there has been a remarkably little advancement in our understanding and treating this annoying condition .

In this study $47.1 \%$ of the patients were over weight this is comparable with the result of prichasuk-S (1994) ${ }^{(1,10)}$ who found $40 \%$ and Turgut-et al (1999) (11) $58 \%$ of their patients to be overweight.

Loss of heel pad elasticity has been suggested as one of the possible explanations of plantar heel pain. Prichasuk-s (1994) $)^{(1)}$ contributed heel pain to heel pad degeneration and he found significant increase in heel pad thickness on standing lateral roentgenograms, while in this study all our patients had heel pad thickness within the normal range despite heel pain, this is same as the finding of Turgut-et al $(1999)^{(11)}$ who found no relation between changes in heel pad elasticity and heel pain when comparing diseased and normal persons and diseased heel with contralateral normal heel in the same patient.

Kumar et al $1979^{(12)}$ proved that plantar heel pain is not due to inflammation in the plantar fascia, but rather a process of degeneration with loss of continuity of collagen fibers and fibroblast infiltration, these changes also found by Singh-et all
All the 12 patients (70.6\%) who were cured had intraosseous pressure values $\geq$ $19 \mathrm{mmHg}$ (mean $21.1 \mathrm{mmHg}$ ), those who failed to respond to surgery in five patients $(29.4 \%)$ all and pressure values of $<19 \mathrm{mmHg}$ (mean $17.7 \mathrm{mmHg}$ ) except one was $19.76 \mathrm{mmHg}$. The nature of pain however has a significant effect on the final outcome all those with throbbing nature of pain showed complete cure $(100 \%)$ while three patients $(37.5 \%)$ of those with searing or pricking pain showed improvement after surgery when considering pain at rest, while both those without night pain failed to respond to surgery. Regarding the presence of a bony spur on x-ray in ten patients $(85.7 \%)$ only six of them showed cure after surgery, while in those patients without spur six out of seven patients $(85.7 \%)$ showed cure after surgery.

$(1997)^{(3)}$ through biopsy specimen from the plantar fascia, so the term plantar fasciitis is a misnomer and the process is actually a degeneration in the proximal plantar fascia and not an inflammation, but this does not imply that it has exact relation to the painful heel as if a biopsy taken from asymptomatic middle age persons the same finding might be found.

The visualization of bony spur on $\mathrm{x}$-ray has no relation with plantar heel pain as in this study $41.2 \%$ of our patients had no spur and $58 \%$ had spur, this is consistent with Barrett-et al $(1999)^{(7)}$ who found no correlation between pain and the presence or absence bony spur, this is also proved by Singh-et al (1997) ${ }^{(3)}$, Gill (1997) ${ }^{(5)}$ and Johal \& Milner $2010^{(8)}$, so the relation between bony spur and painful heel is questionable.

Wilkes was the first who measured normal intraosseous pressure within the calcaneum and described the strong correlation of raised pressure within the cancellous bone and pain, he described a deep boring pain, worse during the night and has been aggravated by physical activity during the day he used the name (intraosseous engorgement pain syndrome), he first described that removal of a plug of bone will relief the pain from this condition ${ }^{(13)}$. 
All these prove that whenever a cancellous bone is exposed to an extraordinary loading and increased stress, substantial increase in intraosseous pressure occur and this increase in pressure ultimately produces what is known as engorgement pain syndrome this is comparable to the condition in plantar heel pain syndrome when the calcaneum which is a cancellous bone exposed to abnormal loading as in athletes, over weight, middle age and jobs with prolonged standing ${ }^{(12)}$.

The major reduction of the patients pain occurred during the first month after surgery although complete disappearance of the pain may take a longer time to occur none of the patients from those who respond to surgery in the first month developed relapse of pain or deterioration during the 6 month follow-up and all are satisfied with results of surgery (12 patients).

Those who are unsatisfied and remained complaining no improvement occurred during the first month of surgery and remained so all over the period.

The intraosseous pressure values in our patients were in a mean of $21.1 \mathrm{mmHg}$ this result was higher than that found by Elshzly $2010^{(14)}$ which was $8 \mathrm{mmHg}$ in normal aclcaneum still we did not consider this to be of great significance because pressure values measured in different studies are not strictly comparable as this influenced by (arterial pressure venous pressure pulse rate, posture, in homogenesity of cancellous bone and the measuring device); this may explain why not all our patients respond to surgery in spite of having high intraosseous pressure than normal but those above $20 \mathrm{mmHg}$ cut off, all are cured so this value and above can be said to be actually pathological those between $19-20 \mathrm{mmHg}$ are variable while those less than $19 \mathrm{mmHg}$ all failed to improve after surgery

Of the five patients who failed to respond to surgery four patients their intraosseous pressure values were below $19 \mathrm{mmHg}$, one has pressure of $19.76 \mathrm{mmHg}$; one of those with pressure values of less than $19 \mathrm{mmHg}$ on postoperative follow-up was found to have tarsal tunnel syndrome on repeating EMG \&NCS.
From this it is, obvious that the preoperative assessment for patient selection is very important to exclude other causes of heel pain, preoperative pressure reading of more than $20 \mathrm{mmHg}$ is associated with a high percentage of cure by surgical decompression with relief improvement of function and early return to job with smooth convalescent period and a few complications. This is in contrary to Brinker et al $1990^{(15)}$ who found significant risk and often prolonged healing and post-operative rehabilitation with even alteration of foot biomechanical integrity after plantar fasciotomy and spur excision these finding also suggested by Levin et al $1998^{(16)}$ who found risks of flattening of medial longitudinal arch and mid foot and lateral foot pain after even partial plantar fascial release.

In this, study $70.6 \%$ patients were satisfied with the final outcome of surgery and they are pain free at 6 month period this is in contrary to Daves-et al (1999) ${ }^{(17)}$ who found only $49 \%$ of his patients satisfied with surgery after plantar fasciotomy and neurolysis of first branch have improvement only to some degree after planter fasciotomy and calcaneal exostectomy (spur excision).

None of the study patients developed fracture of the calcaneum during the operation or afterwards, however Maloniet al $(1992)^{(18)}$ reported in four of his patients calcaneal fracture while walking soon after heel spur excision, this is reported also by Baxter \& Thigpen1984 ${ }^{(19)}$

The modified Maryland foot scoring is a good system for patient selection and its changes after surgery is a good indicator of outcome of surgery, all those patients who got complete, of them cure of symptoms showed the greatest reduction in the scores $(74.8 \%)$ in the first month after surgery, while those still complaining at the end of the study showed minimal reduction of scores $(24.2 \%)$ during the first month, this indicates that although preoperative scores have no relation with the outcome the degree of reduction of it at the first month post operative significantly correlates with the final outcome of surgery. 


\section{CONCLUSION}

- Abnormalities in the intraosseous pressure within the calcaneum are a possible cause of plantar heel pain and can affect the final outcome after calcaneal drilling

- Drilling of the calcaneum is a simple procedure achieving reasonable cure with few complications provided good patient selection was done.

\section{Recommendations}

Conservative treatment of plantar heel pain syndrome should include identification and correction of risk factors calf muscle stretching, lose weight, NSAID, local injection of steroid Surgery is the last resort, and only after all modalities of conservative treatment being tried.

\section{REFRENCES}

1. Prichasuk S.the heel pad in plantar heel pain. J Bone Joint Surg 1994; 76B: 140-142

2. Narvaez JA, Narvaez J,Ortega R; et al. Painful heel: MRI imaging findings. Radiographic 2000; 20: 333-352.

3.Singh D ,Angel J,Bentley G; et al. Fortnightly review: Plantar fasciitis. BMJ 1997; 315: 172-175.

4. Richardson EG: Disorders of tendons and fascia: painful heel. In Canale ST(ed): Campbells operative orthopedics $12^{\text {th }}$ edition, vol 4. St.Loius, Mosby,2012 : 3952-3954.

5. Gill LH Plantar fasciitis :Diagnosis and conservative treatment. J Am Academy Orthop Surg 1997; 5: 109-117.

6. Hassab HK, E1 Sheriff AS. Drilling of the os calcis for painful heel with calcanean spur. Acta Orthop Scand 1974; 45: 152-157.

7. Barrett SJ, O'Malley R.Plantar fasciitis and other causes of heel pain. American family physician. 1999;59(8) 2200-2206.
8. Johal KS, Milner SA. Plantar fasciitis and the calcaneal spur: Fact or fiction Foot and ankle surgery : official journal of the surgeons. 2012; 18(1):39-41.

9. Sadat AM. Plantar fasciitis/calcaneal spur among security forces personnel. Mil Med 1998; 163(1):56-7.

10. Irving DB, Cook JL, Young MA, Menz HB Impact of chronic plantar heel pain on health-related qlity of life. Journal of the American Podiatric Medical Association.2008:Jul.Aug;98(9):283-9.

11. Turgut A, Gokturk E Kose N; et al. The relationship of heel pad elasticity and plantar heel pain. Clin Orthop 1999; 360: 191-196.

12. Kumar S, Davis PR, Pickles B. Bone marrow and bone strength. Acta Orthop Scand 1979; 50: 507-512.

13. Wilkes $\mathrm{CH}$, Visscher $\mathrm{MB}$. Some physiological aspects of bone marrow pressure. J Bone Joint Surg Am 1975; 57: 49-56.

14. El Shazly O,ElBeltagy A. Endoscopic plantar fascia release, calcanedal drilling and calcaneal spur removal for management of painful heel syndrome. Foot (Edinburgh, Scotland). 2010;20(4):121-125.

15. Brinker MR, Lippton HL, Cook SD; et al. Pharmacological regulation of the circulation of bone. J Bone Joint Surg Am 1990; 72:964-975.

16. Levins AD, Skinner HB, Caiozzo VJ. Adaptive gait responses to plantar heel pain. J Rehab Research Development 1998; 35: 289-293.

17. Davies MS, Weiss GA, Saxby TS. Plantar fasciitis: how successful is surgical intervention. Foot-ankle Int 1999 Dec;20(12):803-7.

18. Manoli A, Harper MC, Fitzgibbons TC; et al. Calcaneal fracture after cortical bone removal. Foot Ankle 1992 Nov-Dec; 13(9): 523-5

19. Baxter DE, Thigpen CM. Heel pain: operative results. Foot-Ankle 1984 Jul-Aug; 5(1):16-25. (Abstract) 